\title{
THE GROUP LAW ON A TROPICAL ELLIPTIC CURVE
}

\author{
MAGNUS DEHLI VIGELAND
}

\begin{abstract}
In analogy with the classical group law on a plane cubic curve, we define a group law on a smooth plane tropical cubic curve. We show that the resulting group is isomorphic to $S^{1}$.
\end{abstract}

\section{Introduction}

Tropical geometry is a recent, but rapidly growing field of research in mathematics, in which one seeks to establish connections between complex algebraic geometry and the combinatorics of certain piecewise linear objects, called tropical varieties. Such connections has led to new insight in various areas, like enumerative geometry [3], mirror symmetry [1] and statistics [5].

A favorite subject among many tropical geometers is the study of plane tropical curves, and their many fascinating similarities with classical plane algebraic curves. The purpose of this paper is to give a contribution to the list of such analogies, by defining - in a manner resembling the classical case - a group law on a smooth plane tropical cubic curve.

We define the Jacobian as an abelian group associated to a tropical curve. Unlike the classical situation, the Jacobian of a tropical elliptic curve $C$ is not equal as a set to the curve itself, but to a smaller part of it, namely the curve's unique cycle $\bar{C}$. For $P, Q \in \bar{C}$ we define $d_{C}(P, Q)$ to be the displacement from $P$ to $Q$ with respect to the Z-metric on $\bar{C}$ (and a chosen orientation of $\bar{C})$. This plays a crucial role in the main results, which can be summarized as follows:

THEOREM 1.1. Let $C$ be a tropical elliptic curve, and let $\bar{C}$ be its unique cycle. Let $\mathcal{O}$ be a point on $\bar{C}$.

a) We have a bijection of sets $\bar{C} \longrightarrow \operatorname{Jac}(C)$, given by $P \longmapsto P-\mathcal{O}$.

b) The induced group law on $\bar{C}$ satisfies the relation

$$
d_{C}(\mathscr{O}, P+Q)=d_{C}(\mathscr{O}, P)+d_{C}(\mathscr{O}, Q) .
$$

c) As a group, $\bar{C}$ is isomorphic to the circle group $S^{1}$.

Received October 8, 2007. 


\section{Preliminaries}

Let $R_{\mathrm{tr}}:=(\mathrm{R}, \oplus, \odot)$ be the tropical semiring, where the binary operations are defined by $a \oplus b:=\max \{a, b\}$ and $a \odot b:=a+b$. The multiplicative identity element of $R_{\text {tr }}$ is 0 , while there is no additive identity (since $-\infty$ is not included as an element in $R_{t r}$ ).

REMARK 2.1. The operations $\oplus$ and $\odot$ can be extended to $\mathrm{R}^{n}$ as follows:

$$
\begin{aligned}
\left(a_{1}, \ldots, a_{n}\right) \oplus\left(b_{1}, \ldots, b_{n}\right) & :=\left(\max \left\{a_{1}, b_{1}\right\}, \ldots, \max \left\{a_{n}, b_{n}\right\}\right), \quad \text { and } \\
\lambda \odot\left(a_{1}, \ldots, a_{n}\right) & :=\left(\lambda+a_{1}, \ldots, \lambda+a_{n}\right), \quad \text { for } \lambda \in \mathrm{R} .
\end{aligned}
$$

Moreover, we can define tropical projective $n$-space by setting $\mathrm{P}_{\mathrm{tr}}^{n}:=\mathrm{R}^{n+1} / \sim$, where $\boldsymbol{x} \sim \boldsymbol{y} \Longleftrightarrow \boldsymbol{x}=\lambda \odot \boldsymbol{y}$ for some $\lambda \in \mathrm{R}$. Note that unlike the classical situation, $\mathrm{P}_{\mathrm{tr}}^{n}$ does not have more points than $\mathrm{R}^{n}$. For example, every equivalence class in $\mathrm{P}_{\mathrm{tr}}^{n}$ has a representative in $\mathrm{R}^{n+1}$ with 0 as the last coordinate.

Let $\mathscr{A} \subseteq \mathrm{Z}^{n}$ be a finite set of vectors $\boldsymbol{a}=\left(a_{1}, \ldots, a_{n}\right)$. A tropical (Laurent) polynomial in indeterminates $x_{1}, \ldots, x_{n}$, with support $\mathscr{A}$, is an expression of the form

$$
f=\bigoplus_{\boldsymbol{a} \in \mathscr{A}} \lambda_{\boldsymbol{a}} \odot x_{1}^{a_{1}} \odot \cdots \odot x_{n}^{a_{n}}=\max _{\boldsymbol{a} \in \mathscr{A}}\left\{\ldots, \lambda_{\boldsymbol{a}}+\sum_{i=1}^{n} a_{i} x_{i}, \ldots\right\},
$$

where each $\lambda_{a} \in \mathrm{R}_{\mathrm{tr}}$. The convex hull of $\mathscr{A}$ is called the Newton polytope of $f$ and is denoted by $\Delta$. When in danger of ambiguity, we use indices to indicate the polynomial, as in $\mathscr{A}_{f}$ and $\Delta_{f}$.

Notice that as a function $\mathbf{R}^{n} \rightarrow \mathrm{R}, f$ is convex and piecewise linear.

Definition 2.2. The tropical hypersurface $V(f)$ defined by $f$ is the set of points in $\mathbf{R}^{n}$ where the function $f: \mathbf{R}^{n} \rightarrow \mathrm{R}$ is not linear.

Remark 2.3. Note that if $f$ consists of a single monomial, $V(f)$ is the empty set.

REMARK 2.4. Different tropical polynomials can define the same tropical hypersurface. In particular, it is easy to see that if $g=f \odot m$, where $m=x^{a} y^{b}$ is a tropical monomial, then $V(g)=V(f)$. Note that in this case $\mathscr{A}_{g}$ (resp. $\left.\Delta_{g}\right)$ is a translation of $\mathscr{A}_{f}$ (resp. $\Delta_{f}$ ) by the vector $(a, b)$.

\section{Tropical curves}

We now focus our attention to tropical hypersurfaces in $\mathrm{R}^{2}$ :

Definition 3.1. Let $f(x, y)$ be a tropical polynomial in two indeterminates. The tropical hypersurface $V(f) \subseteq \mathrm{R}^{2}$ is called a tropical curve in $\mathrm{R}^{2}$. 
We recall some basic properties of tropical curves. For proofs and more details, see [6, Section 3], or [3, Sections 1-3] for a more exhaustive approach.

Given a tropical polynomial $f$, we can associate a lattice subdivision of the Newton polygon $\Delta$ of $f$ in the following way: Let $\widehat{\Delta}$ be the convex hull of the set $\left\{\left(a, b, \lambda_{a b c}\right)\right\} \subseteq \mathrm{R}^{2} \times \mathrm{R}$, where $(a, b)$ runs through $\mathscr{A}$. Then define $\operatorname{Subdiv}_{f}$ to be the image under the projection to $R^{2}$ of the top facets of $\widehat{\Delta}$, i.e., the facets whose outer normal unit vector has positive last coordinate.

The subdivision $\operatorname{Subdiv}_{f}$ is in a natural way dual to the tropical hypersurface $V(f)$. In particular, each edge of $V(f)$ corresponds to an edge of $\operatorname{Subdiv}_{f}$, and corresponding edges are perpendicular to each other. The unbounded rays in $V(f)$ correspond to the edges of $\partial \Delta$. (Cf. [6, Proposition 3.5] and [3, Proposition 3.11].)

Let $E$ be an edge of a tropical curve $C=V(f)$, and let $E^{\vee}$ be the corresponding edge in $\operatorname{Subdiv}_{f}$. We define the weight of $E$ to be the lattice length of $E^{\vee}$, i.e. $1+\sharp\left\{\right.$ interior lattice points of $\left.E^{\vee}\right\}$.

LEMma 3.2. For any node $V$ of a tropical curve, the following balancing condition holds: Let $E_{1}, \ldots, E_{n}$ be the edges adjacent to $V$. For each $i=$ $1, \ldots, n$ let $m_{i}$ be the weight of $E_{i}$, and $\boldsymbol{v}_{i}$ the primitive integer vector pointing into $E_{i}$ from $V$. Then

$$
m_{1} \boldsymbol{v}_{1}+\cdots+m_{n} \boldsymbol{v}_{n}=\mathbf{0}
$$

where $\mathbf{0}=(0,0) \in \mathbf{R}^{2}$.

The balancing condition characterizes tropical curves: Assume $C$ is a 1dimensional polyhedral complex in $\mathrm{R}^{2}$, consisting of rays and line segments with rational slopes, each assigned some positive integral weight. Then $C=$ $V(f)$ for some tropical polynomial $f$ if and only if (1) is satisfied at every vertex of $C$.

Next we define the degree of a tropical curve. For each $d \in \mathrm{N}_{0}$, let $\Gamma_{d}$ be the triangle with vertices $(0,0),(d, 0),(0, d)$. (When $d=0$ we get the degenerated triangle $\Gamma_{0}=\{(0,0)\}$.)

Definition 3.3. Let $C=V(f)$ be a tropical curve in $\mathrm{R}^{2}$, and let $\Delta$ be the Newton polygon of $f$. If $\Delta$ fits inside $\Gamma_{d}$, but not inside $\Gamma_{d-1}$, then $C$ has degree $d$. If $\Delta=\Gamma_{d}$, we say that $C$ has degree $d$ with full support.

Remark 3.4. There seems to be no clear consensus in the literature on how to define the degree of a tropical curve. Definition 3.3 differs slightly from the ones in [6] and [3], but serves the purpose of this paper better. In particular, as we will see in the next section, Definition 3.3 gives room for an extended version of the tropical Bezout's theorem compared to that in [6]. 
ExAmPLE 3.5. A tropical line is a tropical curve of degree 1 with full support. For instance, if $f=a x \oplus b y \oplus c$, then the tropical line $L=V(f)$ consists of three unbounded rays, emanating from the "center" $(c-a, c-b)$ in the directions $(-1,0),(0,-1)$ and $(1,1)$ respectively.

Example 3.6. If $f$ is any monomial, then $\Delta$ consists of a single point. Hence $V(f)$ has degree 0 . This is appropriate since $V(f)$ is an empty set. (Cf. Remark 2.3.)

A vertex $V$ of a tropical curve is called 3 -valent if $V$ has exactly 3 adjacent edges. Furthermore, if these edges have weights $m_{1}, m_{2}, m_{3}$ and primitive integer direction vectors $\boldsymbol{u}=\left(u_{0}, u_{1}\right), \boldsymbol{v}=\left(v_{0}, v_{1}\right), \boldsymbol{w}=\left(w_{0}, w_{1}\right)$ respectively, we define the multiplicity of $V$ to be the absolute value of the number

$$
m_{1} m_{2}\left|\begin{array}{cc}
u_{0} & u_{1} \\
v_{0} & v_{1}
\end{array}\right|=m_{2} m_{3}\left|\begin{array}{cc}
v_{0} & v_{1} \\
w_{0} & w_{1}
\end{array}\right|=m_{1} m_{3}\left|\begin{array}{cc}
w_{0} & w_{1} \\
u_{0} & u_{1}
\end{array}\right| .
$$

Definition 3.7. A tropical curve is called smooth if every vertex is 3 -valent and has multiplicity 1 .

Notice that in a smooth tropical curve, every edge has weight 1.

Definition 3.8. The genus of a smooth tropical curve $C=V(f)$ is the number of vertices of $\operatorname{Subdiv}_{f}$ in the interior of the Newton polygon $\Delta_{f}$.
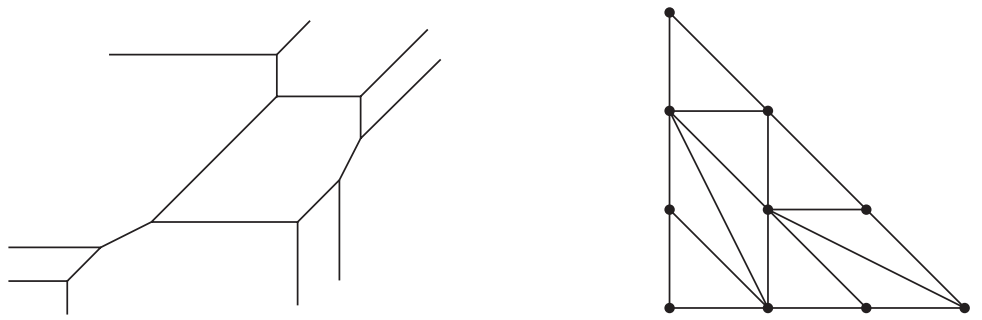

Figure 1. A tropical curve and its associated subdivision. The subdivision shows that the curve is smooth of degree 3 and genus 1 .

Figure 1 shows a smooth curve of degree 3 and genus 1, and its associated subdivision.

\subsection{The Z-metric}

Let $C \subseteq \mathrm{R}^{2}$ be a smooth tropical curve. If $E$ is any edge of $C$, we define a metric on $E$ called the Z-metric, in the following way. For any two points $\boldsymbol{x}, \boldsymbol{y} \in E$, we set their distance in the Z-metric to be the number $\frac{\|\boldsymbol{x}-\boldsymbol{y}\|}{\|\boldsymbol{v}\|}$, where $\|\cdot\|$ denotes the Euclidean norm, and $v$ is a primitive integral direction vector of $E$. In particular, if $E$ is a bounded edge, we define its lattice length, $l(E)$, to be 
the distance (in the Z-metric) between its endpoints. Note that if both endpoints of $E$ have integral coordinates, then $l(E)=1+\sharp$ interior lattice points on $E\}$.

REMark 3.9. By identifying each edge $E$ of $C$ with the real interval $[0, l(E)]$ (or $[0, \infty)$ if $E$ is unbounded), $C$ can be thought of as a "metric graph with possibly unbounded edges". This is equivalent to giving $C$ a Zaffine structure, or tropical structure as described e.g. in [4].

\section{Intersections of tropical curves}

We say that two tropical curves $C$ and $D$ intersect transversally if no vertex of $C$ lies on $D$ and vice versa. In a transversal intersection we define intersection multiplicities as follows: Let $P$ be an intersection point of $C$ and $D$, where the two edges meeting have weights $m_{1}$ and $m_{2}$, and primitive direction vectors $\left(v_{0}, v_{1}\right)$ and $\left(w_{0}, w_{1}\right)$ respectively. The intersection multiplicity mult ${ }_{P}(C \cap D)$ is then the absolute value of

$$
m_{1} m_{2}\left|\begin{array}{cc}
v_{0} & v_{1} \\
w_{0} & w_{1}
\end{array}\right| .
$$

Non-transversal intersections are dealt with in the following way: For any intersecting tropical curves $C$ and $D$, let $C_{\varepsilon}$ and $D_{\varepsilon}$ be nearby translations of $C$ and $D$ such that $C_{\varepsilon}$ and $D_{\varepsilon}$ intersect transversally. We then have ([6, Theorem 4.3]):

Theorem-Definition 4.1. Let the stable intersection of $C$ and $D$, denoted $C \cap_{\text {st }} D$, be defined by

$$
C \cap_{\mathrm{st}} D=\lim _{\varepsilon \rightarrow 0}\left(C_{\varepsilon} \cap D_{\varepsilon}\right) .
$$

This limit is independent of the choice of perturbations, and is a well-defined subset of points with multiplicities in $C \cap D$.

Theorem 4.2 (Tropical Bezout). Assume $C$ and $D$ are tropical curves of degrees $c$ and $d$ respectively. If both curves have full support, then their stable intersection consists of $c d$ points, counting multiplicities.

Proof. See [6, Theorem 4.2 and Corollary 4.4]. The idea is to show that the number of (stable) intersection points is invariant under translations of the curves. Thus we can arrange the two curves such that for each of them, the intersection points lie on the unbounded rays in one of the three coordinate directions. It is then trivial to check that $\sharp\left(C \cap_{\mathrm{st}} D\right)=c d$.

There is also a tropical version of Bernstein's Theorem: Recall that the mixed area of two convex polygons $R$ and $S$ is defined as the number Area $(R+S)-$ $\operatorname{Area}(R)-\operatorname{Area}(S)$, where $R+S$ is the Minkowski sum of $R$ and $S$. 
Theorem 4.3 (Tropical Bernstein). Let $C=V(f)$ and $D=V(g)$ be any tropical curves intersecting transversally, with Newton polygons $\Delta_{f}$ and $\Delta_{g}$ respectively. Then the number of intersection points, counting multiplicities, equals the mixed area of $\Delta_{f}$ and $\Delta_{g}$.

Proof. See [7, Theorem 9.5].

Although perhaps not as enlightening as the homotopy argument given in [6], one can prove Theorem 4.2 as a special case of Theorem 4.3. In fact, we can get a stronger result:

Theorem 4.4 (Strong version of Tropical Bezout). Assume $C$ and $D$ are tropical curves of degrees $c$ and $d$ respectively. If at least one of the curves have full support, then their stable intersection consists of cd points, counting multiplicities.

Proof. Because of Theorem-Definition 4.1 we can assume that the intersection is transversal. Note that for any positive integers $c$ and $d$, we have the Minkowski sum $\Gamma_{c}+\Gamma_{d}=\Gamma_{c+d}$. Hence the mixed area of $\Gamma_{c}+\Gamma_{d}$ equals $\frac{1}{2}(c+d)^{2}-\frac{1}{2} c^{2}-\frac{1}{2} d^{2}=c d$. This proves Theorem 4.2.

Suppose now $C$ has full support, i.e. $\Delta_{f}=\Gamma_{c}$, and that $\Delta_{g}$ is a convex polygon of the form $\Gamma_{d} \backslash Q$, where $Q \subseteq \Gamma_{d}$ is a lattice polygon containing exactly one of the corners of $\Gamma_{d}$, say $(d, 0)$. Then Area $\left(\Delta_{f}+\Delta_{g}\right)=\operatorname{Area}\left(\Gamma_{c}+\right.$ $\left.\left(\Gamma_{d} \backslash Q\right)\right)=\operatorname{Area}\left(\Gamma_{c}+\Gamma_{d}\right)-\operatorname{Area}(Q)$. Thus the mixed area of $\Delta_{f}$ and $\Delta_{g}$ is

$$
\begin{aligned}
& \operatorname{Area}\left(\Delta_{f}+\Delta_{g}\right)-\operatorname{Area}\left(\Delta_{f}\right)-\operatorname{Area}\left(\Delta_{g}\right) \\
& \quad=\left(\operatorname{Area}\left(\Gamma_{c}+\Gamma_{d}\right)\right)-\operatorname{Area}(Q)-\operatorname{Area}\left(\Gamma_{d}\right)-\left(\operatorname{Area}\left(\Gamma_{d}\right)-\operatorname{Area}(Q)\right) \\
& \quad=c d .
\end{aligned}
$$

The same argument shows that we can do the same at the other corners, without changing the mixed area. In this way we can form any Newton polygon $\Delta_{g}$ associated to a tropical curve of degree $d$. Hence $\sharp\left(C \cap_{\mathrm{st}} D\right)=c d$ for any tropical curve $D$ of degree $d$.

Remark 4.5. If neither of the two curves have full support, the theorem will not hold in general. For example, if $C$ and $D$ are the quadric curves given by $C=V\left(x^{2} \oplus y\right)$ and $D=V\left(x \oplus y^{2}\right)$, then $C \cap D$ consists of a single point with multiplicity 3 . Another example is given by the non-intersecting lines $V(0 \oplus x)$ and $V(1 \oplus x)$.

An important special case of Theorem 4.4 is the following corollary:

Corollary 4.6. Let $D$ be any tropical curve of degree $d$. Then any tropical line meets $D$ stably in exactly d points, counting multiplicities. 


\section{Divisors on smooth tropical curves}

Let $C$ be a smooth tropical curve in $\mathrm{R}^{2}$.

Definition 5.1. We define the group of divisors on $C, \operatorname{Div}(C)$, to be the free abelian group generated by the points on $C$. A divisor $D$ on $C$ is an element of $\operatorname{Div}(C)$, i.e. a finite formal sum of the form $D=\sum \mu_{P} P$.

The number $\sum \mu_{P}$ is as usual called the degree of $D$. Observe that the elements of degree 0 in $\operatorname{Div}(C)$ form a group, denoted by $\operatorname{Div}^{0}(C)$.

To define principle divisors, we must first define rational functions. By a tropical rational function $h: \mathrm{R}^{2} \rightarrow \mathrm{R}$ we mean a function of the form $h=f-g$, where $f$ and $g$ are tropical polynomials with equal Newton polygons.

Definition 5.2. Given a tropical polynomial $f$, we define the divisor $\operatorname{div}(f) \in \operatorname{Div}(C)$ as the formal sum of points in $C \cap_{\text {st }} V(f)$, counted with their respective intersection multiplicities. Furthermore, if $h=f-g$ is a tropical rational function on $\mathrm{R}^{2}$, we set $\operatorname{div}(h):=\operatorname{div}(f)-\operatorname{div}(g)$. A divisor $D \in \operatorname{Div}(C)$ is called a principal divisor if $D=\operatorname{div}(h)$ for some tropical rational function $h$.

It follows from Theorem 4.3 that any principal divisor on $C$ has degree 0 .

Remark 5.3. Suppose the Newton polygons of $f$ and $g$ differ by a translation. Then we would still have $\operatorname{div}(f)-\operatorname{div}(g) \in \operatorname{Div}^{0}(C)$, because of Theorem 4.3. In fact, $\operatorname{div}(f)-\operatorname{div}(g)$ is a principle divisor. Indeed, if $(a, b)$ is the translation vector from $\Delta_{f}$ to $\Delta_{g}$, let $m=x^{a} y^{b}$ be the corresponding tropical monomial. Since $V(f)=V(f \odot m)$ (by Remark 2.4) it follows that $\operatorname{div}(f)-\operatorname{div}(g)=\operatorname{div}(h)$, where $h$ is the tropical rational function $(f \odot m)-g$.

Definition 5.4. Two divisors $D_{1}$ and $D_{2}$ are linearly equivalent, denoted as $D_{1} \sim D_{2}$, if $D_{1}-D_{2}$ is principal.

Linear equivalence is an equivalence relation, and as in the classical case one can show that it restricts to an equivalence relation on the subgroup $\operatorname{Div}^{0}(C)$. Hence we can make the following definition:

Definition 5.5. The group $\operatorname{Div}^{0}(C) / \sim$ is called the Jacobian of $C, \operatorname{Jac}(C)$.

\subsection{A formula for the divisor of a tropical rational function}

The purpose of this section is to develop a formula for the divisor of a tropical rational function $h: \mathrm{R}^{2} \rightarrow \mathrm{R}$, using only the properties of $h$ restricted to $C$. We begin with some easy observations:

Lemma 5.6. Let $h: \mathrm{R}^{2} \rightarrow \mathrm{R}$ be a tropical rational function, and $C \subseteq \mathrm{R}^{2}$ a tropical curve. The restriction of $h$ to $C$ is then 
a) continuous on $C$,

b) piecewise linear on each edge of $C$, with integer slopes (with respect to the Z-metric on the edge),

c) eventually constant on each unbounded ray of $C$.

Proof. a) Note that $h$ is the difference of tropical polynomials, which are continuous.

b) It is enough to prove this for tropical polynomials. Let $E$ be an edge of $C$, with primitive integer direction vector $\boldsymbol{v}$, and let $f(\boldsymbol{x})=\max _{\boldsymbol{a} \in \mathscr{A}}\left\{\alpha_{\boldsymbol{a}}+\right.$ $\boldsymbol{a} \cdot \boldsymbol{x}\}$ be a tropical polynomial function. Clearly, $\left.f\right|_{E}$ is piecewise linear on $E$. Furthermore, consider any point $P \in E$ such that $f$ is linear in an open interval $I \subseteq E$ containing $P$. Then $f(\boldsymbol{x})=\alpha_{\boldsymbol{a}}+\boldsymbol{a} \cdot \boldsymbol{x}$ for all $\boldsymbol{x} \in I$, for some $\boldsymbol{a} \in \mathscr{A}$, and the slope of $f$ at $P$ in the direction of $\boldsymbol{v}$ (w.r.t the Z-metric on $E$ ), is $f(P+\boldsymbol{v})-f(P)=\boldsymbol{a} \cdot \boldsymbol{v} \in \mathbf{Z}$.

c) Suppose $h(\boldsymbol{x})=f(\boldsymbol{x})-g(\boldsymbol{x})$, where $f(\boldsymbol{x})=\max _{\boldsymbol{a} \in \mathscr{A}_{f}}\left\{\alpha_{\boldsymbol{a}}+\boldsymbol{a} \cdot \boldsymbol{x}\right\}$ and $g(\boldsymbol{x})=\max _{\boldsymbol{b} \in \mathscr{A}_{g}}\left\{\beta_{\boldsymbol{b}}+\boldsymbol{b} \cdot \boldsymbol{x}\right\}$ are tropical polynomials with $\Delta_{f}=\Delta_{g}$. Let $\ell=\{V+t \boldsymbol{u} \mid t \geq 0\}$ be an unbounded ray of $C$, starting at the vertex $V$ and with primitive direction vector $\boldsymbol{u}$. Then $f(V+t \boldsymbol{u})=\max _{\boldsymbol{a} \in \mathscr{A}_{f}}\left\{\alpha_{\boldsymbol{a}}+\boldsymbol{a} \cdot V+t(\boldsymbol{a} \cdot \boldsymbol{u})\right\}$. For all $t \gg 0$ this maximum is achieved for some $\boldsymbol{a}=\boldsymbol{a}_{\ell}$ with the property that $\boldsymbol{a}_{\ell} \cdot \boldsymbol{u} \geq \boldsymbol{a} \cdot \boldsymbol{u}$ for all $\boldsymbol{a} \in \mathscr{A}_{f}$. In particular this implies that $\boldsymbol{a}_{\ell} \in \partial \Delta_{f}$. Similarly, when $t \gg 0$, we have $g(V+t \boldsymbol{u})=\beta_{\boldsymbol{b}_{\ell}}+\boldsymbol{b}_{\ell} \cdot V+t\left(\boldsymbol{b}_{\ell} \cdot \boldsymbol{u}\right)$, for some $\boldsymbol{b}_{\ell} \in \partial \Delta_{g}$ such that $\boldsymbol{b}_{\ell} \cdot \boldsymbol{u} \geq \boldsymbol{b} \cdot \boldsymbol{u}$ for all $\boldsymbol{b} \in \mathscr{A}_{g}$. Since $\Delta_{f}=\Delta_{g}$ this implies that $\boldsymbol{a}_{\ell} \cdot \boldsymbol{u}=\boldsymbol{b}_{\ell} \cdot \boldsymbol{u}$, and we conclude that for $t \gg 0$ we have $h(V+t \boldsymbol{u})=\alpha_{\boldsymbol{a}_{\ell}}-\beta_{\boldsymbol{b}_{\ell}}+\left(\boldsymbol{a}_{\ell}-\boldsymbol{b}_{\ell}\right) \cdot V$, which is constant.

For any function $r: C \rightarrow$ R satisfying a) and b) above, we associate to each point $P \in C$ an integer $\operatorname{ord}_{P}(r)$ as follows: If $P$ is a vertex of $C$, then $\operatorname{ord}_{P}(r)$ is the sum of the outgoing slopes of $r$ along the edges adjacent to $P$. If $P \in C$ is not a vertex, we use the same definition, after first having inserted a (2-valent) vertex at $P$ (but otherwise keeping $C$ unchanged).

We say that $r$ is locally linear at $P \in C$ if there exists an open neighborhood $U \subseteq \mathrm{R}^{2}$ containing $P$, and an affine-linear function $s: \mathrm{R}^{2} \rightarrow \mathrm{R}$ such that $\left.r\right|_{C \cap U}=\left.s\right|_{C \cap U}$. It is easy to see that $\operatorname{ord}_{P}(r)=0$ if $r$ is locally linear at $P$. Note however, that the converse is not true if $P$ is a vertex of $C$.

We are now ready to prove the following:

Lemma 5.7. For any tropical rational function $h: \mathrm{R}^{2} \rightarrow \mathrm{R}$ we have

$$
\operatorname{div}(h)=\sum_{P \in C} \operatorname{ord}_{P}\left(\left.h\right|_{C}\right) P .
$$

Proof. Let $f=\max _{\boldsymbol{a} \in \mathscr{A}_{f}}\left\{\alpha_{\boldsymbol{a}}+\boldsymbol{a} \cdot \boldsymbol{x}\right\}$ be a tropical polynomial, and let $\bar{f}:=\left.f\right|_{C}$. It is clear that $\bar{f}$ is locally linear at any $P \in C \backslash\left(C \cap_{\mathrm{st}} V(f)\right)$, 
and therefore $\operatorname{ord}_{P}(\bar{f})=0$ for such $P$. We show below that for each $P \in$ $C \cap_{\mathrm{st}} V(f)$, the intersection multiplicity at $P$ equals $\operatorname{ord}_{P}(\bar{f})$. This implies $\operatorname{div}(f)=\sum_{P \in C} \operatorname{ord}_{P}(\bar{f}) P$. By the obvious extension from tropical polynomials to tropical rational functions, the lemma follows from this.

Consider first the case where $P$ is a transversal intersection point, between an edge $E_{C}$ of $C$ and an edge $E_{f}$ of $V(f)$. We can choose primitive direction vectors $\boldsymbol{v}=\left(v_{1}, v_{2}\right)$ and $\boldsymbol{u}=\left(u_{1}, u_{2}\right)$ of $E_{C}$ and $E_{f}$ respectively, such that if $m$ is the weight of $E_{f}$, the intersection multiplicity is mult $_{P}(C \cap V(f))=$ $m\left(v_{1} u_{2}-v_{2} u_{1}\right)$.

To find $\operatorname{ord}_{P}(\bar{f})$, suppose $\boldsymbol{a}, \boldsymbol{b} \in \mathscr{A}_{f}$ are such that $f$ equals $\alpha_{\boldsymbol{a}}+\boldsymbol{a} \cdot \boldsymbol{x}$ on one side of $P$, and $\alpha_{\boldsymbol{b}}+\boldsymbol{b} \cdot \boldsymbol{x}$ on the other side. Then $\boldsymbol{b}-\boldsymbol{a}$ is orthogonal to $E_{f}$. Moreover, by the definition of weight, we have (possibly after swapping $\boldsymbol{a}$ and $\boldsymbol{b})$ that $\boldsymbol{b}-\boldsymbol{a}=m\left(u_{2},-u_{1}\right)$. This implies that $\operatorname{ord}_{P}(\bar{f})=\boldsymbol{v} \cdot \boldsymbol{b}+(-\boldsymbol{v}) \cdot \boldsymbol{a}=$ $\boldsymbol{v} \cdot(\boldsymbol{b}-\boldsymbol{a})=m\left(v_{1} u_{2}-v_{2} u_{1}\right)$, which equals the intersection multiplicity found above.

Next, suppose $P \in C \cap_{\text {st }} V(f)$ is a non-transversal intersection point, i.e., that $P$ is a vertex of either $C$ or $V(f)$. In either case, consider $f_{\varepsilon}=$ $\max _{\boldsymbol{a} \in \mathscr{A}_{f}}\left\{\alpha_{\boldsymbol{a}}^{\epsilon}+\boldsymbol{a} \cdot \boldsymbol{x}\right\}$ such that $V\left(f_{\varepsilon}\right)$ is a small translation of $V(f)$ intersecting $C$ transversally, and $P \notin V\left(f_{\varepsilon}\right)$. Let $P_{1}, \ldots, P_{k} \in C \cap V\left(f_{\varepsilon}\right)$ be the intersection points close to $P$ (i.e. the points tending to $P$ when $f_{\varepsilon} \rightarrow f$ ). Then we have

$$
\operatorname{mult}_{P}\left(C \cap_{\mathrm{st}} V(f)\right)=\sum_{i=1}^{k} \operatorname{mult}_{P_{i}}\left(C \cap V\left(f_{\varepsilon}\right)\right) .
$$

We proceed to show that $\operatorname{ord}_{P}(\bar{f})$ shows a similar, stable behavior. Let $\ell_{1}, \ldots, \ell_{s}$ be the edges of $C$ emanating from $P$, with primitive direction vectors $\boldsymbol{v}_{1}, \ldots, \boldsymbol{v}_{s}$. (If $P$ is not a vertex of $C$, we insert a vertex at $P$, making $s=2$, and $v_{1}=-v_{2}$.) Furthermore, let $\boldsymbol{a}_{1}, \ldots, \boldsymbol{a}_{s} \in \mathscr{A}_{f}$ be such that for $\boldsymbol{x} \in \ell_{i}$ close to $P$, we have $f(\boldsymbol{x})=\lambda_{\boldsymbol{a}_{i}}+\boldsymbol{a}_{i} \cdot \boldsymbol{x}$. In particular, with this notation, we have $\operatorname{ord}_{P}(\bar{f})=\sum_{i=1}^{s} \boldsymbol{a}_{i} \cdot \boldsymbol{v}_{i}$.

Because $P \notin V\left(f_{\varepsilon}\right), f_{\varepsilon}$ is locally linear at $P$, and we can assume w.l.o.g. that $f_{\varepsilon}(\boldsymbol{x})=\alpha_{a_{1}}^{\varepsilon}+\boldsymbol{a}_{1} \cdot \boldsymbol{x}$ in a neighborhood of $P$. For $j=1, \ldots, s$, let $B_{j} \subseteq\left\{P_{1}, \ldots, P_{k}\right\}$ be the subset whose elements lies on $\ell_{j}$. It is not hard to see that if $B_{j} \neq \emptyset$, then $\sum_{Q \in B_{j}} \operatorname{ord}_{Q}\left(\bar{f}_{\varepsilon}\right)=\boldsymbol{a}_{j} \cdot \boldsymbol{v}_{j}-\boldsymbol{a}_{1} \cdot \boldsymbol{v}_{j}$. Hence,

$$
\begin{aligned}
\sum_{i=1}^{k} \operatorname{ord}_{P_{i}}\left(\bar{f}_{\varepsilon}\right) & =\sum_{B_{j} \neq \emptyset}\left(\boldsymbol{a}_{j} \cdot \boldsymbol{v}_{j}-\boldsymbol{a}_{1} \cdot \boldsymbol{v}_{j}\right) \\
& =\sum_{B_{j} \neq \emptyset} \boldsymbol{a}_{j} \cdot \boldsymbol{v}_{j}+\sum_{B_{j}=\emptyset} \boldsymbol{a}_{1} \cdot \boldsymbol{v}_{j}=\operatorname{ord}_{P}(\bar{f}),
\end{aligned}
$$


where in the second to last transition we used the balancing condition, and in the final transition the easily proved observation that if $B_{j}=\emptyset$ then $\boldsymbol{a}_{j}=\boldsymbol{a}_{1}$.

From (2) and (3) we deduce that $\operatorname{mult}_{P}\left(C \cap_{\mathrm{st}} V(f)=\operatorname{ord}_{P}(\bar{f})\right.$ also when $P$ is non-transversal, and hence that $\operatorname{div}(f)=\sum \operatorname{ord}_{P}(\bar{f}) P$. This proves the lemma.

Remark 5.8. A consequence of the above two lemmas is that our definitions of tropical rational functions and their divisors are in agreement with those used by Gathmann and Kerber in [2]. The set $\mathscr{R}=\left\{\left.h\right|_{C} \mid h\right.$ is a tropical rational function $\}$ is a subset of what they call rational functions on $C$, i.e. functions $r: C \rightarrow \mathrm{R}$ which satisfy parts a) and b) of Lemma 5.6. Moreover, Lemma 5.7 implies that for any function in $\mathscr{R}$, the definition of its associated divisor given in [2] is equivalent to our Definition 5.2. In particular, the endpoints at infinity of unbounded rays (these are included as part of the curve in [2]) are avoided because of Lemma 5.6c).

\section{Tropical elliptic curves}

In the remainder of the paper $C$ will denote a tropical elliptic curve, by which we mean a smooth tropical curve of degree 3 and genus 1 . We assume that $C=V(f)$, where $f(x, y)$ has Newton polygon $\Delta_{f} \subseteq \Gamma_{3}$. Since $(1,1)$ is the only lattice point in the interior of $\Gamma_{3}$, the definition of genus requires that $(1,1)$ is a vertex of $\operatorname{Subdiv}_{f}$ lying in the interior of $\Delta_{f}$. Hence $C$ contains a unique cycle, which we will denote by $\bar{C}$. Finally, each connected component of $C \backslash \bar{C}$ is called a tentacle of $C$.

\subsection{An explicit homeomorphism $\bar{C} \rightarrow S^{1}$}

Obviously, as a topological space, $\bar{C}$ is homeomorphic to the circle group $S^{1}$. We will now construct one such homeomorphism, based on the Z-metric on the edges of $C$.

Choose any fixed point $\mathcal{O} \in \bar{C}$. Let $V_{1}, \ldots, V_{n}$ be the vertices of $\bar{C}$ in counter-clockwise direction, such that if $\mathscr{O}$ is a vertex then $V_{1}=\mathscr{O}$, otherwise $\mathscr{O}$ lies between $V_{1}$ and $V_{n}$. Let $E_{1}, \ldots, E_{n}$ be the edges of $\bar{C}$, such that $E_{1}=$ $V_{1} V_{2}$ and so on. Recall that for each $i, l\left(E_{i}\right)$ denotes the length of $E_{i}$ in the Z-metric on $E_{i}$. Let $l$ be the cycle length of $\bar{C}$, i.e., $l=l\left(E_{1}\right)+\cdots+l\left(E_{n}\right)$.

We now define a homeomorphism $\mu: \bar{C} \longrightarrow \mathrm{R} / \mathrm{lZ} \approx S^{1}$, linear in the Euclidean metric of each edge $E_{i}$. It is then enough to specify the images in $\mathrm{R} / l Z$ of the points $\mathcal{O}, V_{1}, \ldots, V_{n}$, which we do recursively:

$$
\begin{aligned}
\mu(\mathscr{O}) & =0 \\
\mu\left(V_{1}\right) & =l\left(\mathscr{O} V_{1}\right) \\
\mu\left(V_{i+1}\right) & =\mu\left(V_{i}\right)+l\left(E_{i}\right), \quad i=1, \ldots, n-1 .
\end{aligned}
$$


Finally, identifying $\mathrm{R} / l \mathrm{Z}$ with the interval $[0, l)$, we define the (signed) displacement function $d_{C}: \bar{C} \times \bar{C} \rightarrow \mathrm{R}$ by the formula

$$
d_{C}(P, Q)=\mu(Q)-\mu(P) .
$$

Note that $d_{C}(Q, P)=-d_{C}(P, Q)$ for any $P, Q \in \bar{C}$. Moreover, for any three points $P, Q, R \in \bar{C}$ we have $d_{C}(P, Q)+d_{C}(Q, R)=d_{C}(P, R)$.

\subsection{When are two points on C linearly equivalent?}

In this section we give two propositions, which together give a complete answer to the question in the title. Namely, we prove that any two points on the same tentacle are linearly equivalent, while two distinct points on $\bar{C}$ are never linearly equivalent.

Proposition 6.1. Let $P$ and $Q$ be points on the same tentacle of $C$. Then $P \sim Q$.

Proof. We begin by showing that the points on any unbounded ray are equivalent. By symmetry, it is enough to prove this for the rays that are unbounded in, say, the $x$-coordinate. Figure 2 shows a typical situation with three such rays, $\ell_{1}, \ell_{2}$ and $\ell_{3}$.
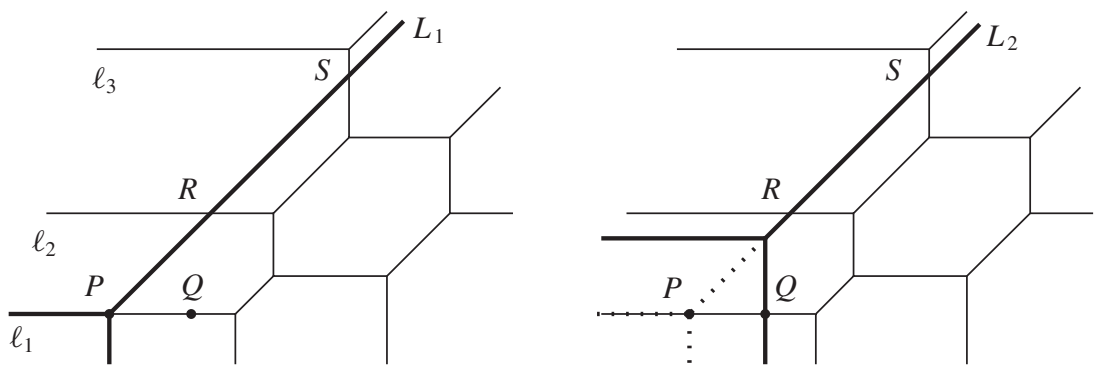

FIGURE 2. Sufficiently close points $P$ and $Q$ on $\ell_{1}$ are linearly equivalent.

The following argument shows that any two sufficiently close points $P$ and $Q$ on $\ell_{1}$ are equivalent: Assume $P$ is further away from $\bar{C}$ than $Q$. Let $h=f_{1}-f_{2}$ be the tropical rational function where $f_{1}$ and $f_{2}$ are tropical linear polynomials such that $L_{1}=V\left(f_{1}\right)$ is the tropical line with center in $P$, and $L_{2}=V\left(f_{2}\right)$ is the line passing through $Q$ and with center on the ray of $L_{1}$ with direction vector $(1,1)$. Denote this ray by $\rho$. Then $\operatorname{div}\left(f_{1}\right)=P+R+S$, where $R$ and $S$ lies on $\rho$, and $\operatorname{div}\left(f_{2}\right)=Q+R+S$ (as long as $P$ and $Q$ are close enough). It follows that $\operatorname{div}(h)=P-Q$, in other words $P \sim Q$.

To show that any two points $P$ and $Q$ on $\ell_{1}$ are equivalent, we can choose a finite sequence of points $P=P_{1}, P_{2}, \ldots, P_{m}=Q$ on $\ell_{1}$ such that each pair 


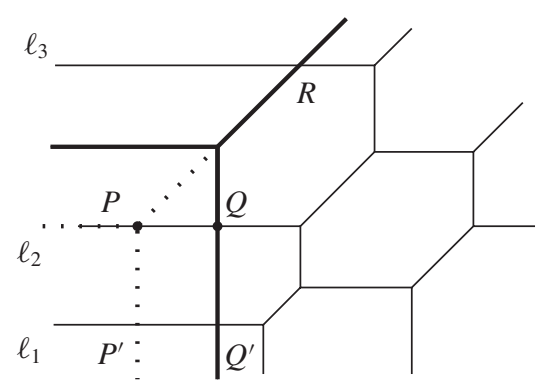

Figure 3. $P \sim Q$ on $\ell_{2}$.

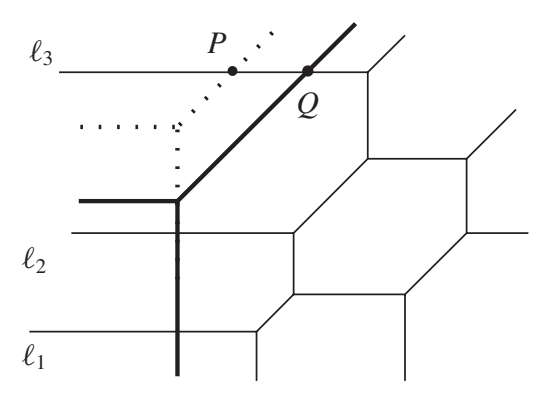

Figure 4. $P \sim Q$ on $\ell_{3}$.

$\left(P_{i}, P_{i+1}\right)$ is close enough for the above technique to work. Then $P=P_{1} \sim$ $\cdots \sim P_{m}=Q$.

A similar argument shows that the points on $\ell_{2}$ are equivalent. The idea is sketched in Figure 3. To show that $P$ and $Q$ are equivalent, take the tropical line $L_{1}$ with center in $P$ and slide it along the ray with direction $(1,1)$ (i.e. keeping $R$ as intersection point with $C$ ) until it passes through $Q$. With the notation on Figure 3, we see that $P+P^{\prime}+R \sim Q+Q^{\prime}+R$. But $P^{\prime} \sim Q^{\prime}$, since they are on $\ell_{1}$, thus $P \sim Q$.

The same technique works for $\ell_{3}$ (see Figure 4) and also for the bounded line segments of the tentacles. Any tentacle of a tropical elliptic curve can be handled in this way.

Proposition 6.2. If $P, Q \in \bar{C}$ and $P \sim Q$, then $P=Q$.

Proof. Suppose otherwise that $P \neq Q$, and that there exists a tropical rational function $h$ such that $\operatorname{div}(h)=P-Q$. We will apply Lemma 5.7 to show that this leads to a contradiction.

Let $\bar{h}=\left.h\right|_{C}$. As a first observation, note that $\bar{h}$ is constant on each tentacle of $C$. Indeed, this follows from Lemma 5.6c) and the fact that $\operatorname{ord}_{R}(\bar{h})=0$ for all points $R \in C \backslash \bar{C}$. (Note in particular that if $\bar{h}$ is constant on two edges adjacent to a 3 -valent vertex $V$, then $\operatorname{ord}_{V}(\bar{h})=0$ implies that $\bar{h}$ is constant on the third edge as well.)

Now, let $c_{1}$ and $c_{2}$ be the two directed polygonal arcs of $\bar{C}$ from $P$ to $Q$. We claim that for each $i=1,2, \bar{h}$ has constant slope along $c_{i}$, w.r.t. the Z-metric. To see this, observe that $\bar{h}$ is clearly linear along any edge of $c_{i}$. Furthermore, suppose two edges of $c_{i}$ intersect in a vertex $V \in \bar{C}$, and that the slopes of $\bar{h}$ along these edges (directed from $P$ to $Q$ ) are $s_{1}$ and $s_{2}$. Because $\bar{h}$ is constant on the tentacle adjacent to $V$, $\operatorname{ord}_{V}(\bar{h})$ is of the form $\pm\left(s_{1}-s_{2}+0\right)$. This equals 0 , hence $s_{1}=s_{2}$. This proves the claim. Since $\operatorname{ord}_{P}(\bar{h})=1$, the slopes of $\bar{h}$ along the paths $c_{1}$ and $c_{2}$ must be $s$ and $1-s$ for some $s \in$ Z. But this contradicts the assumption of continuity of $\bar{h}$ at $Q$, since for any choice of $s$, 


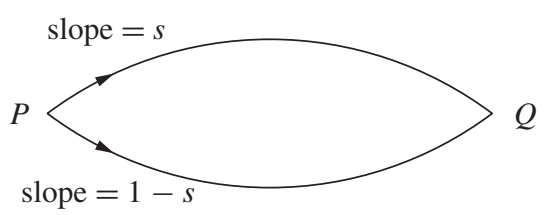

FIGURE 5. Slope properties of a function $h$ satisfying $\operatorname{div}(h)=P-Q$.

one of the numbers $s$ and $1-s$ is positive, while the other is non-positive. (See Figure 5.)

\subsection{The group law}

In this final section we show that the $\operatorname{Jacobian} \operatorname{Jac}(C)$ is set-theoretically equal to $\bar{C}$, and describe the resulting group structure on $\bar{C}$. A crucial step towards this goal is to determine when divisors of the form $P+Q$ are linearly equivalent. When trying to imitate the techniques from the classical case, we stumble across the following problem: Given two points $P$ and $Q$ on $\bar{C}$, we cannot always find a tropical line $L$ that intersects $C$ stably in $P$ and $Q$. (Recall that a stable intersection is defined as a limit of transversal intersections.) If there exists such a tropical line, we call $(P, Q)$ a good pair.

We fix the notation $\boldsymbol{p}_{1}=(-1,0), \boldsymbol{p}_{2}=(0,-1)$ and $\boldsymbol{p}_{3}=(1,1)$ for the primitive integer direction vectors of a tropical line.

Lemma 6.3. Let $P, Q, P^{\prime}, Q^{\prime}$ be any points on $\bar{C}$. Then

$$
P+Q \sim P^{\prime}+Q^{\prime} \Longleftrightarrow d_{C}\left(P, P^{\prime}\right)=-d_{C}\left(Q, Q^{\prime}\right) .
$$

Proof. We proceed in two steps. First, we prove the result when $(P, Q)$ and $\left(P^{\prime}, Q^{\prime}\right)$ are good pairs. Using this, we then generalize to any pairs.

- Step 1. Assume $(P, Q)$ and $\left(P^{\prime}, Q^{\prime}\right)$ are good pairs, and that $P+Q \sim$ $P^{\prime}+Q^{\prime}$. Then there exists (unique) tropical lines $L$ and $L^{\prime}$, and a point $R \in \bar{C}$ such that $L \cap_{\mathrm{st}} C=P+Q+R$ and $L^{\prime} \cap_{\mathrm{st}} C=P^{\prime}+Q^{\prime}+R$. (Note that the existence of $R$ follows from Proposition 6.2.) Consider a homotopy $L_{t}$ of lines containing $R$ such that $L_{0}=L$ and $L_{1}=L^{\prime}$. It is enough to consider the case where $P$ and $P^{\prime}$ are on the same edge, $Q$ and $Q^{\prime}$ are on the same edge, and $L^{\prime}$ is a parallel displacement of $L$ along one of the axes. Indeed, in more complex cases, the homotopy can be broken down into parts with the above properties. 


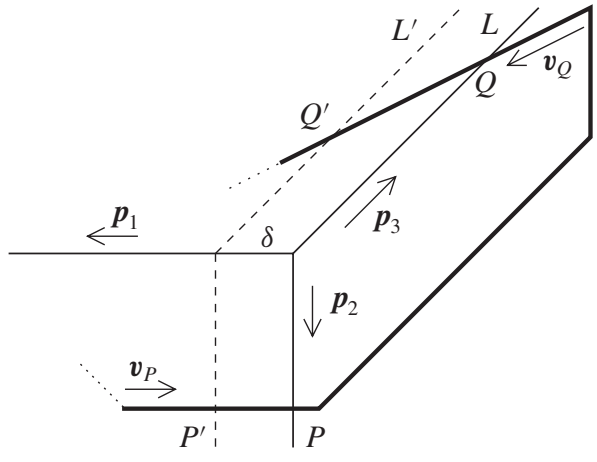

Figure 6. Step 1.

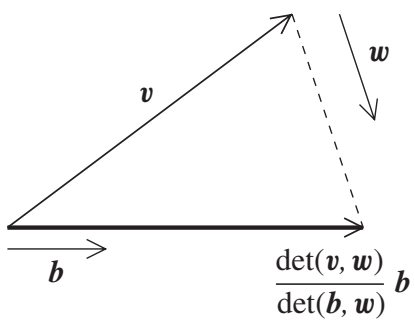

FIGURE 7. Non-orthogonal projection in $R^{2}$.

Let $\boldsymbol{v}_{P}$ and $\boldsymbol{v}_{Q}$ be primitive integer direction vectors of the edges of $\bar{C}$ containing $P, P^{\prime}$ and $Q, Q^{\prime}$ respectively, and assume that $L^{\prime}$ equals the shifting of $L \delta$ units in the direction of, say, $\boldsymbol{p}_{1}$ (see Figure 6). Then from the formula for nonorthogonal vector projection (Figure 7), we find the Z-metric displacements of $P$ and $Q$ :

$$
\begin{aligned}
& \left|d_{C}\left(P, P^{\prime}\right)\right|=\frac{\left\|P^{\prime}-P\right\|}{\left\|\boldsymbol{v}_{P}\right\|}=\frac{\left\|\frac{\operatorname{det}\left(\delta \boldsymbol{p}_{1}, \boldsymbol{p}_{2}\right)}{\operatorname{det}\left(\boldsymbol{v}_{P}, \boldsymbol{p}_{2}\right)} \boldsymbol{v}_{P}\right\|}{\left\|\boldsymbol{v}_{P}\right\|}=|\delta|, \quad \text { and } \\
& \left|d_{C}\left(Q, Q^{\prime}\right)\right|=\frac{\left\|Q^{\prime}-Q\right\|}{\left\|\boldsymbol{v}_{Q}\right\|}=\frac{\left\|\frac{\operatorname{det}\left(\delta \boldsymbol{p}_{1}, \boldsymbol{p}_{3}\right)}{\operatorname{det}\left(\boldsymbol{p}_{Q}, \boldsymbol{p}_{3}\right)} \boldsymbol{v}_{Q}\right\|}{\left\|\boldsymbol{v}_{Q}\right\|}=|\delta|
\end{aligned}
$$

(Note that $\left|\operatorname{det}\left(\boldsymbol{v}_{P}, \boldsymbol{p}_{2}\right)\right|=\left|\operatorname{det}\left(\boldsymbol{v}_{Q}, \boldsymbol{p}_{3}\right)\right|=1$ since the corresponding intersections have multiplicity 1.) Finally, it is clear that $P$ and $Q$ are moved in opposite directions on $\bar{C}$. Hence $d_{C}\left(P, P^{\prime}\right)=-d_{C}\left(Q, Q^{\prime}\right)$ as claimed.

The implication $\Leftarrow$ follows by a similar argument.

- Step 2. Now assume $(P, Q)$ is not a good pair. Let $L_{1}$ and $L_{2}$ be tropical lines through $P$ and $Q$ respectively, and let $R_{1}, S_{1}, R_{2}, S_{2}$ be the other intersection points. The idea is to move $L_{1}$ and $L_{2}$ into new lines $L_{1}^{\prime}$ and $L_{2}^{\prime}$ in such a way that $R_{1}, S_{1}, R_{2}, S_{2}$ are preserved as intersection points. $P$ and $Q$ are not preserved; they move to the two new intersection points $P^{\prime}$ and $Q^{\prime}$ (see Figure 8). By construction, these points satisfy $P^{\prime}+Q^{\prime} \sim P+Q$. Using our results in Step 1 on each of the lines $L_{1}$ and $L_{2}$, it follows that $d_{C}\left(P, P^{\prime}\right)=-d_{C}\left(Q, Q^{\prime}\right)$. Conversely, it is not hard to see that in this way one can reach any nearby pair $\left(P^{\prime}, Q^{\prime}\right)$ satisfying $d_{C}\left(P, P^{\prime}\right)=-d_{C}\left(Q, Q^{\prime}\right)$.

Moving $P$ and $Q$ far enough apart (sometimes more than one move is needed) one obtains a good pair $\left(P^{\prime}, Q^{\prime}\right)$. Since we proved in Step 1 that the 

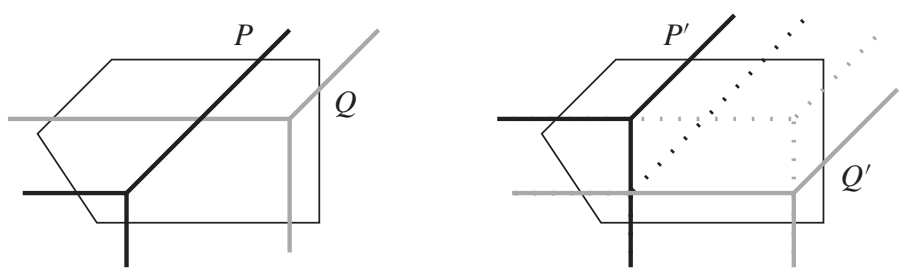

Figure 8. Moving a bad pair $(P, Q)$.

lemma holds for good pairs, it follows that the lemma is true for any pairs $(P, Q)$ and $\left(P^{\prime}, Q^{\prime}\right)$.

Proposition 6.4. For any fixed point $\mathcal{O} \in \bar{C}$, the map $\tau_{\mathcal{O}}: \bar{C} \rightarrow \operatorname{Jac}(C)$ given by $P \mapsto P-\mathscr{O}$ is a bijection of sets.

Proof. Injectivity follows immediately from Lemma 6.3, since

$$
P-\mathscr{O} \sim Q-\mathscr{O} \Longrightarrow P+\mathscr{O} \sim Q+\mathscr{O} \Longrightarrow d_{C}(P, Q)=0 \Longrightarrow P=Q \text {. }
$$

To prove surjectivity, let $D$ be any divisor of degree 0 . We must show that there exists $P \in \bar{C}$ such that $D \sim P-\mathcal{O}$. Assume first that $D=P_{1}-Q_{1}$, where $P_{1}, Q_{1} \in \bar{C}$. Choose $P$ such that $d_{C}\left(P, P_{1}\right)=d_{C}\left(\mathscr{O}, Q_{1}\right)$, then Lemma 6.3 gives $P+Q_{1} \sim P_{1}+\mathscr{O}$. Thus we have $D=P_{1}-Q_{1} \sim P-\mathscr{O}$.

Now assume $D=D_{1}-D_{2}$, where $D_{1}=P_{1}+\cdots+P_{n}$ and $D_{2}=$ $Q_{1} \cdots+Q_{n}$ are any effective divisors of degree $n>1$. Let $P_{12}$ and $Q_{12}$ be points such that $P_{1}+P_{2} \sim \mathscr{O}+P_{12}$ and $Q_{1}+Q_{2} \sim \mathscr{O}+Q_{12}$. Then

$$
\begin{aligned}
D & \sim \mathcal{O}+P_{12}+\cdots+P_{n}-\left(\mathscr{O}+Q_{12}+\cdots+Q_{n}\right) \\
& =P_{12}+\cdots+P_{n}-\left(Q_{12}+\cdots+Q_{n}\right) .
\end{aligned}
$$

Hence $D \sim D_{1}^{\prime}-D_{2}^{\prime}$, where $D_{1}$ and $D_{2}$ are effective of degree $n-1$. This way we can reduce to the case $n=1$, which we already proved.

Because of Proposition 6.4, $\bar{C}$ has a natural group structure:

Definition 6.5. Define $(\bar{C}, \mathscr{O})$ to be the group consisting of points on $\bar{C}$, with the group structure induced from $\operatorname{Jac}(C)$ such that the bijection $\tau_{\mathcal{O}}$ is an isomorphism of groups.

The next theorem and its corollary are the main results of this paper.

Theorem 6.6. Let $P$ and $Q$ be any points on $\bar{C}$, and let + denote addition in the group $(\bar{C}, \mathcal{O})$. Then the point $P+Q$ satisfies the relation

$$
d_{C}(\mathcal{O}, P+Q)=d_{C}(\mathscr{O}, P)+d_{C}(\mathscr{O}, Q) .
$$


Proof. Because $\tau_{\mathscr{O}}$ is a group isomorphism, the following equalities hold in $\operatorname{Jac}(C)$ :

$$
(P+Q)-\mathscr{O}=\tau_{\mathscr{O}}(P+Q)=\tau_{\mathscr{O}}(P)+\tau_{\mathscr{O}}(Q)=P-\mathscr{O}+Q-\mathscr{O} .
$$

Thus in $\operatorname{Jac}(C)$ we have $(P+Q)+\mathscr{O}=P+Q$. This means that the divisors $(P+Q)+\mathscr{O}$ and $P+Q$ are equivalent, which by Lemma 6.3 implies the relation

$$
d_{C}(P, P+Q)=d_{C}(\mathscr{O}, Q) .
$$

Adding $d_{C}(\mathscr{O}, P)$ on each side then gives $d_{C}(\mathscr{O}, P+Q)=d_{C}(\mathscr{O}, P)+$ $d_{C}(\mathcal{O}, Q)$ as wanted.

Remark 6.7. We can describe the group law geometrically just as in the classical case of elliptic curves: To add $P$ and $Q$ we do the following. If $(P, Q)$ is a good pair, consider the tropical line $L$ through $P$ and $Q$, and let $R$ be the third intersection point of $L$ and $\bar{C}$. Now if $(R, \mathscr{O})$ is a good pair, let $L^{\prime}$ be the through $R$ and $\mathcal{O}$. Then $P+Q$ is the third intersection point of $L^{\prime}$ and $\bar{C}$. (See Figure 9 for an example.)
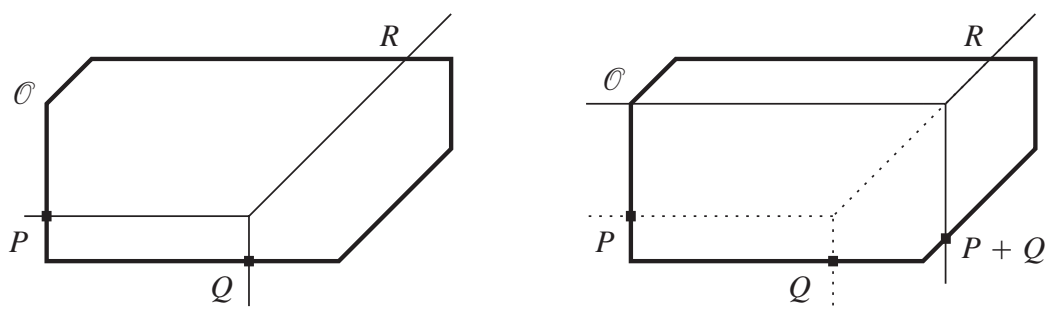

FIGURE 9. Adding points on a tropical elliptic curve.

If any of the pairs $(P, Q)$ and $(R, \mathscr{O})$ fail to be good, then move the two points involved equally far (in the Z-metric) in opposite directions until they form a good pair, and use this new pair as described above.

Corollary 6.8. The map $\mu:(\bar{C}, \mathscr{O}) \longrightarrow \mathrm{R} / l \mathrm{Z} \approx S^{1}$ defined in (4) is a group isomorphism.

Proof. It follows from the relation (5) that for any $P$ we have $\mu(P)=$ $d_{C}(\mathscr{O}, P)$. Thus

$$
\mu(P+Q)=d_{C}(\mathscr{O}, P+Q)=d_{C}(\mathscr{O}, P)+d_{C}(\mathscr{O}, Q)=\mu(P)+\mu(Q) .
$$

ACKNOWLEDGement. Thanks to Grigory Mikhalkin for helpful advice at an early stage of this work, and to Kristian Ranestad for many useful discussions, suggestions and corrections. 


\section{REFERENCES}

1. Boehm, J., Mirror symmetry and tropical geometry, preprint, arxiv:math.AG/0708.4402, 2007.

2. Gathmann, A., and Kerber, M.,A Riemann-Roch theorem in tropical geometry, Math. Z. 259 (2007), 217-230.

3. Mikhalkin, G.,Enumerative tropical algebraic geometry in $\mathrm{R}^{2}$, J. Amer. Math. Soc. 18 (2005), 313-377.

4. Mikhalkin, G., and Zharkov, I., Tropical curves, their Jacobians and Theta functions, preprint, arXiv:math.AG/0612267, 2006.

5. Pachter, L., and Sturmfels, B., Tropical geometry of statistical models, Proc. Natl. Acad. Sci. USA 101 (2004), 16132-16137.

6. Richter-Gebert, J., Sturmfels, B., and Theobald, T., First steps in tropical geometry, pp. 289317 in: Idempotent Mathematics and Mathematical Physics, Contemp. Math. 377, Amer. Math. Soc., Providence, RI 2005.

7. Sturmfels, B., Solving Systems of Polynomial Equations, CBMS Regional Conference Series in Mathematics 97, published for the Conference Board of the Mathematical Sciences, Amer. Math. Soc., Providence, RI 2002.

DEPARTMENT OF MATHEMATICS

UNIVERSITY OF OSLO

NORWAY

E-mail:magnusv@math.uio.no 